\title{
Target Material Irradiation Studies for High-Intensity Accelerator Beams
}

\author{
N. Simos ${ }^{1 *}$, H. Kirk ${ }^{1}$, H. Ludewig ${ }^{1}$, P. Thieberger ${ }^{1}$, W-T Weng ${ }^{1}$, K. McDonald ${ }^{2}$, J. Sheppard ${ }^{3}$, G. Evangelakis ${ }^{4}$, \\ and K. Yoshimura \\ ${ }^{1}$ Brookhaven National Laboratory, UPTON, NY 11973, USA \\ ${ }^{2}$ Princeton University, Princeton, NJ, USA \\ ${ }^{3}$ Stanford Linear Accelerator, Stanford, CA, USA \\ ${ }^{4}$ University of Ioannina, Department of Physics, Ioannina, Greece \\ ${ }^{5}$ KEK, Tsukuba, Japan
}

This paper presents results of recent experimental studies focusing on the behavior of special materials and composites under irradiation conditions and their potential use as accelerator targets. The paper also discusses the approach and goals of on-going investigations on an expanded material matrix geared toward the neutrino superbeam and muon collider initiatives.

\section{BACKGROUND}

With increasing demand for high-power accelerators in support of initiatives for new physics opportunities such as the neutrino superbeam and the muon collider/neutrino factory, where intense secondary beams of pions, muons and neutrinos are of great interest, the pool of materials that can be utilized and play the role of production targets is dramatically reduced. At power levels of $1 \mathrm{MW}$ and higher the development of integrated target systems that can function under such demand is a serious challenge due to uncertainties associated with the long-term survival of the highly irradiated target material. The ever greater deposited energy and induced thermo-mechanical loads combined with irradiation are the reason for the limitations.

To overcome the challenges and meet the physics requirements, an intensive search has been under way for both "smart" target designs and target materials that exhibit favorable behavior over specific ranges of key physical or mechanical properties that define their resilience. While many materials are well understood from their application to other areas, most of them are screened-out due to their inability to withstand the level of shock anticipated. A variety of new and "smart" materials have recently become available to serve the needs of special industries and some of them hold the promise of playing the role of high power targets while eliminating the primary failure mode. The advantage of these materials, as it applies to high power targets, stems primarily from the low thermal expansion they exhibit and secondly from the increased strength.

During the BNL E951 experiment [1], typical graphite (ATJ-grade) thermo-mechanical response to rapid proton heating was compared to the response of a carbon-carbon (CC) composite which is specially fabricated to eliminate thermal expansion and thus eliminate thermal stress. Figure 1 depicts recorded strains in the two types of targets intercepting $24 \mathrm{GeV}$ proton pulses. The superiority of the new composite is clearly shown. However, the question of whether this superior response of the composite holds true when is irradiated is still unanswered. Irradiation studies are needed to confirm its resilience to such levels of exposure.

The goal of the study is to qualitatively assess how prone are these "new" material to experiencing degradation of their advantageous properties under irradiation. This experimental assessment is only a qualitative evaluation (due to low cumulative irradiation levels that are feasible at this stage). Candidate materials that show promise will be included in a follow-up phase of irradiation to levels of displacement per atom (dpa) that are anticipated during the useful life of the target.

Several studies have been performed in recent years [2, 3] (in addition to the BNL E951) that addressed the properties of several materials that are

\footnotetext{
* Work performed under the auspices of the US DOE
} 
either in use or planned to be used in accelerator components. These include the Accelerator Production of Tritium (APT) Material Handbook study and the Spallation Neutron Source studies in the US, Europe and Japan. While an extensive matrix of materials has been used (between the various studies), no information regarding irradiation effects on the new alloys/composites is available. It should be noted that the wealth of reactor-based material irradiation experience continues to provide the baseline for the new studies for long-term irradiation exposure extrapolation, understanding mechanisms of material property changes, etc.

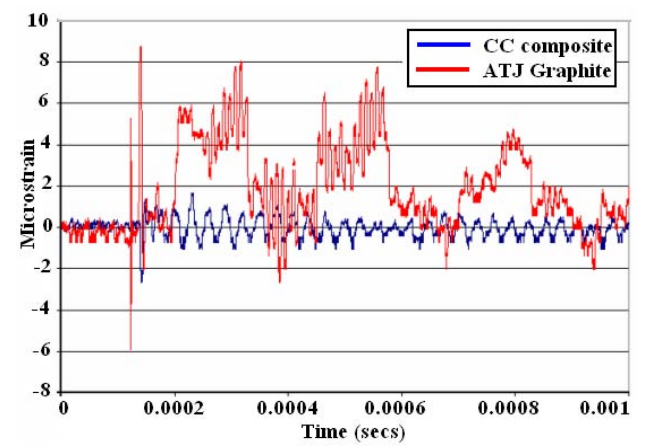

Figure 1: Graphite vs. Carbon-Carbon composite response (strain) to $24 \mathrm{GeV}$ Protons

\section{MATERIAL IRRADIATION STUDY}

\subsection{Phase I}

During Phase-I of the material irradiation study attention was primarily focused on Super-Invar and in a lesser degree on Inconel-718. Relevant information to the overall study was also obtained from its predecessor (BNL Experiment E951) where several materials (either in the form of proton windows and targets) were subjected to $24 \mathrm{GeV}$ proton pulses and had their dynamic response measured and analyzed. These materials included Al-3000, Inconel-718, Havar and Ti alloy (6Al-6V) as windows and graphite (ATJ grade) and CC composite as low-Z targets. It was indicated earlier that the CC composite appears to minimize the thermal shock as compared to classical graphite. Super-Invar held the same promise based on its low CTE over a specific range of operating temperature.

\subsection{Phase II}

A number of new "smart" alloys and composites other than CC composite and super-Invar have been developed in recent years for a wide range of applications. These materials exhibit either high strength or, as in the case of Invar, low CTE. Such a situation appears to be beneficial to the accelerator community since it optimizes the two material properties that matter the most. For example, Figure 3 depicts the characterization of the "Gum Metal" alloy (Ti based alloy) that seems to have significant advantages over other alloys. However, these alloys/composites were never evaluated for radiation damage. Their properties are, nevertheless, intriguing enough to consider them for high-power target applications, provided that the radiation damage issues are addressed.

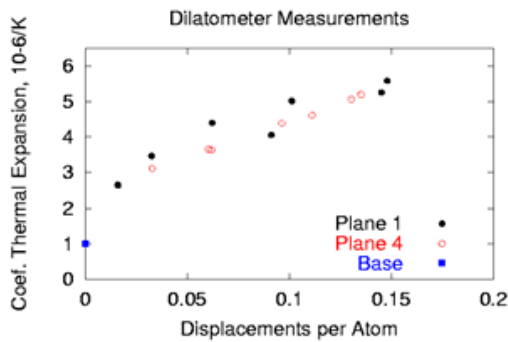

Figure 2: Irradiation effects on CTE of super-Invar.

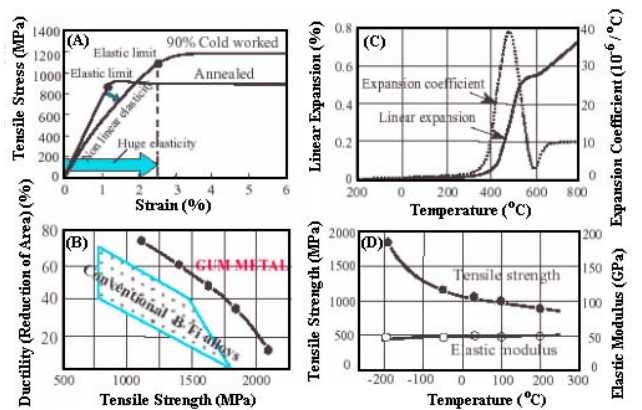

Figure 3: "Gum Metal" material characterization in its non-irradiated state [4]

During Phase-II of the material study the selected matrix of materials has been irradiated with $200 \mathrm{MeV}$ protons and average current of $70 \mu \mathrm{A}$ at the BNL BLIP facility for two weeks. The exposure is expected to induce approximately $0.25 \mathrm{dpa}$ in the higher $\mathrm{Z}$ materials of the matrix. Figure 4 depicts the two types of specimens (tensile and CTE) as well as their typical assembly in one of the irradiation boxes. The adopted configuration allowed for multiple layers of specimens and cooling channels for the forced water flow through the assembly. The stacking configuration was a requirement so that the Gaussian beam profile is preserved upon exiting the 
irradiated material. Below is the list of the materials selected for irradiation.

- Carbon-Carbon composite. A three-dimensional weaved fiber structure with good strength and very low $\mathrm{CTE}$ up to about $1000^{\circ} \mathrm{C}$. The minimization of the thermal shock in the target material is the key driver.

- Ti-6Al-4V. Titanium alloy combining good tensile strength and relatively low CTE.

- Toyota "Gum Metal". Alloy with ultra-low elastic modulus, high strength, super-elastic like nature and near-zero CTE in the range of -200 ${ }^{\circ} \mathrm{C}$ to $+250{ }^{\circ} \mathrm{C}$.

- VASCOMAX. High strength alloy considered as high-Z target. Irradiation effects on CTE, fracture toughness and ductility loss not known.

- AlBeMet. A new, low-Z alloy combining the properties of Be and Al. Irradiation effects on its properties need to be assessed.

- Beryllium. Assessment of its resilience as a beam window material for the muon collider.

- Graphite IG-43. A promising graphite grade with good strength but no irradiation history.

- Nickel-plated Aluminum. Baseline material for the focusing horn of the neutrino beam experiments (NUMI, BNL Superbeam, etc.). Bonding degradation due to irradiation and water environment to be assessed.

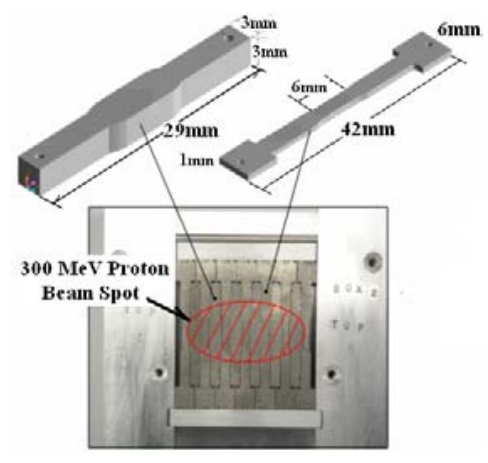

Figure 4: BNL BLIP Irradiation specimen assembly.

\section{IRRADIATION STUDY RESULTS}

Irradiation of the selected material matrix was completed in March of 2004. While the irradiated specimens are "cooling-down" for post-irradiation studies in the BNL hot cell facility starting in November of 2004, the irradiation temperature was addressed with a separate experiment at the BNL
BLIP using Temperature Sensitive Paint and the same configuration as in the actual irradiation box. Figure 5 depicts some of the results.
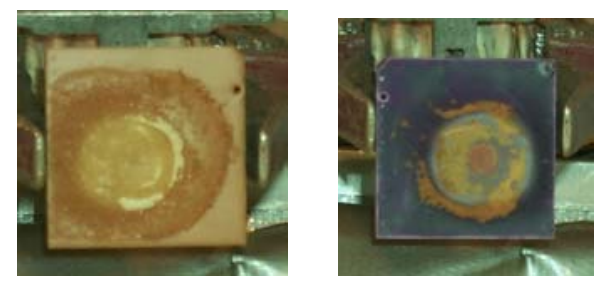

Figure 5: Experimental verification of irradiation temperature using Thermal Sensitive Paint (TSP)

Radiographic analysis of thin nickel films embedded in the specimen array has also been performed to establish the beam profile. Some of the results are shown in Figure 6. Specimen activation analysis has also been performed. Results of postirradiation analysis on physical and mechanical property including activation will soon be reported.

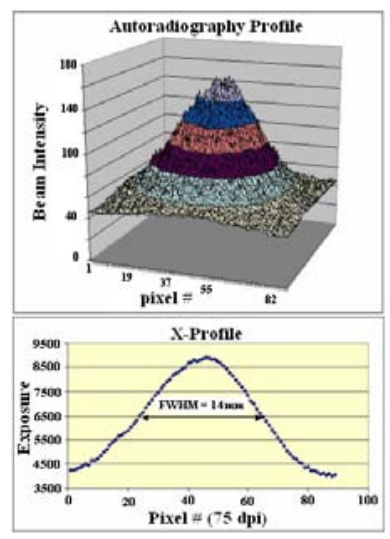

Figure 6: Beam spot radiographic analysis.

\section{REFERENCES}

1. H.G. Kirk, et.al., "Target Studies with BNL E951 at the AGS," Proc. 2001 PAC, Chicago, Il., March 2001, p.1535.

2. M. Kawai, K. Kikuchi, editors, "Proc. of $3^{\text {rd }}$ Workshop on Material Science and Technology for Spallation Neutron Source", KEK Proc. 2003-6, 2002

3. APT Materials Handbook, TPO-P00-MDDX-00001, 20001.

4. K. Nishino, "Super Multifunctional Alloy "GUM METAL".Toyota CRDL., INC. Technical News. 